\title{
Erratum: Effects of a chiral three-nucleon force on nucleus-nucleus scattering [Phys. Rev. C 90, 051601(R) (2014)]
}

\author{
K. Minomo, M. Toyokawa, M. Kohno, and M. Yahiro \\ (Received 6 June 2017; published 27 November 2017)
}

DOI: 10.1103/PhysRevC.96.059904

As reported in the Erratum [1], several errors were found in the program code for the nuclear-matter calculation reported in the associated original paper [2]. The changes in the $G$ matrices in nuclear matter including the three-nucleon force (3NF) effects affect the results of subsequent applications of them to the description of nucleus-nucleus scatterings reported in the original paper for which we present corrected results in the present Erratum.

The single-particle potentials in Fig. 1 in the original paper are corrected as shown in Fig. 1 for each spin-isospin channel. The $3 \mathrm{NF}$ effects become weaker except for the real part in the ${ }^{3} \mathrm{O}$ channel.

The real and imaginary parts of the ${ }^{16} \mathrm{O}-{ }^{16} \mathrm{O}$ potential at $70 \mathrm{MeV} /$ nucleon are revised as shown in Fig. 2. Corresponding to the change in $3 \mathrm{NF}$ effects shown in Fig. 1, the $3 \mathrm{NF}$ effects on the folding potential become somewhat weaker.

The corrected angular distributions of the ${ }^{16} \mathrm{O}-{ }^{16} \mathrm{O}$ and ${ }^{12} \mathrm{C}-{ }^{12} \mathrm{C}$ scatterings are shown in Fig. 3. On the whole, the

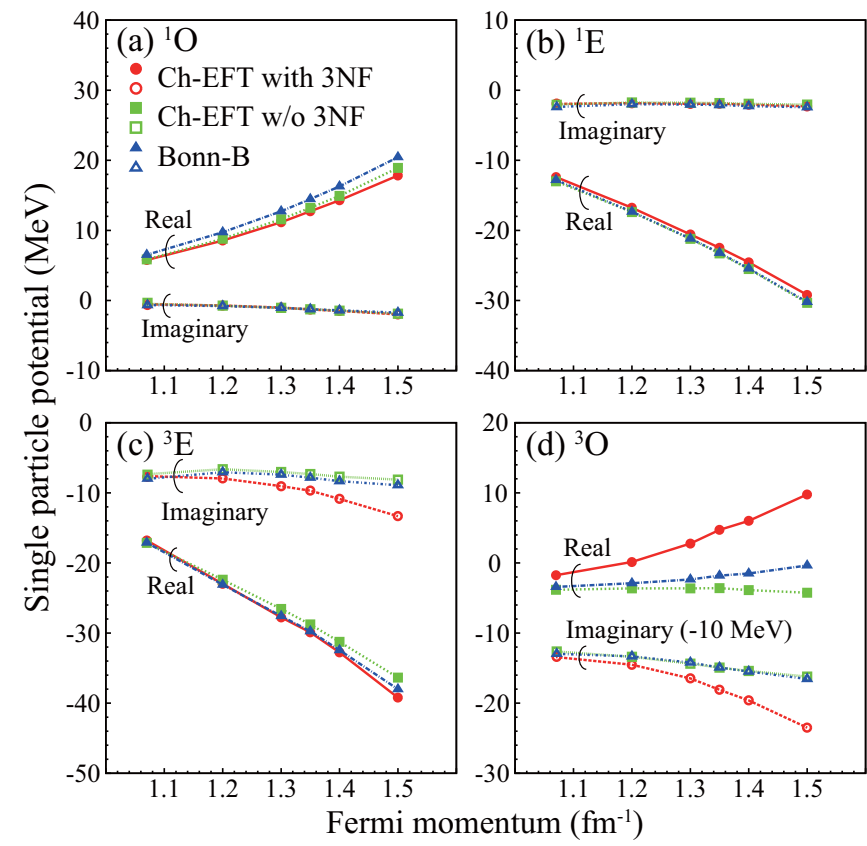

FIG. 1. Revised version of Fig. 1 in the original paper. $k_{F}$ dependence of the nucleon single-particle potential in each spinisospin channel at $E=70 \mathrm{MeV}$ : (a) ${ }^{1} \mathrm{O}$, (b) ${ }^{1} \mathrm{E}$, (c) ${ }^{3} \mathrm{E}$, and (d) ${ }^{3} \mathrm{O}$ channels. The circles, squares, and triangles stand for the results of the chiral two-nucleon force $(2 \mathrm{NF})+3 \mathrm{NF}$, chiral $2 \mathrm{NF}$, and Bonn-B $2 \mathrm{NF}$, respectively. The closed (open) symbols correspond to the real (imaginary) part of the single-particle potentials. For ${ }^{3} \mathrm{O}$, the imaginary part is shifted down by $10 \mathrm{MeV}$.
3NF effects persist, improving the correspondence between the calculated and the measured cross sections, although the agreement is insufficient at large angles. It has been confirmed that the $3 \mathrm{NF}$ effects on the total reaction cross sections are still small. Our conclusion for the $3 \mathrm{NF}$ effects in the nucleus-nucleus elastic scatterings does not change qualitatively.
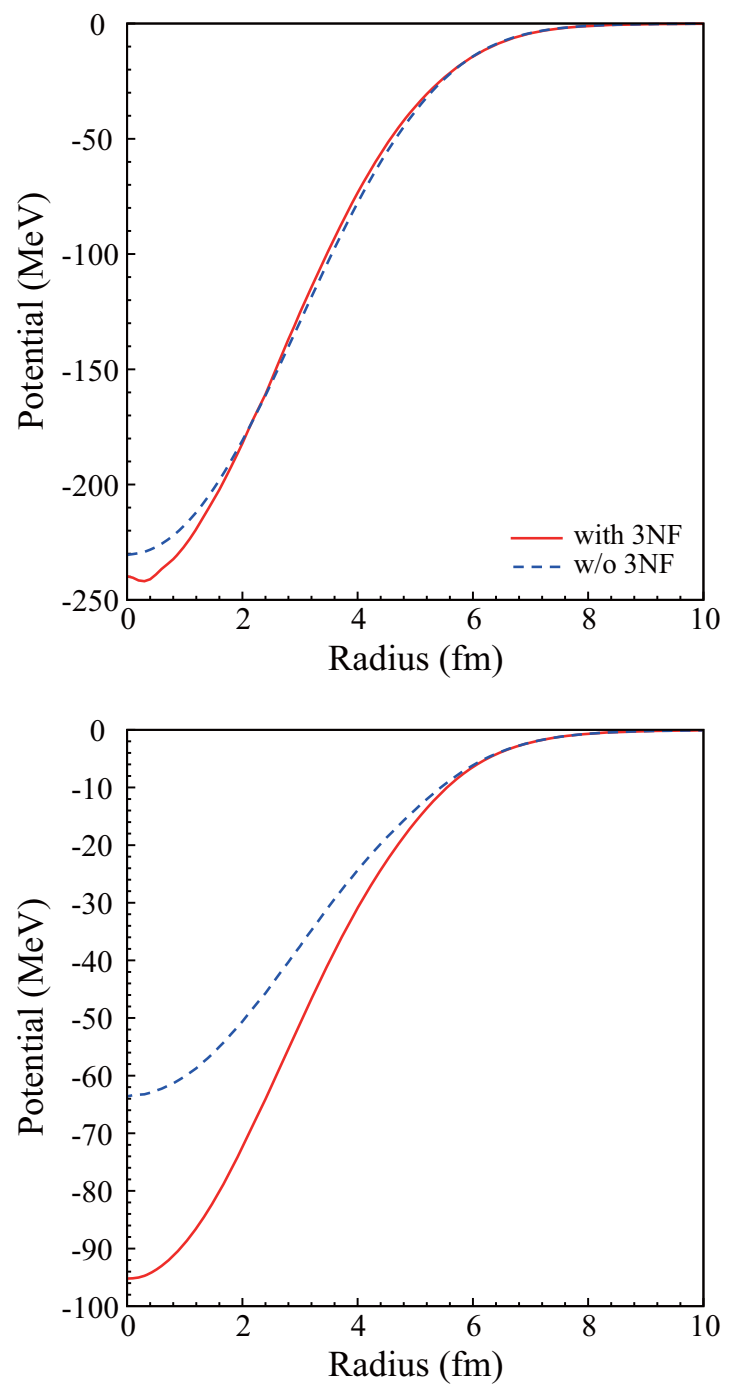

FIG. 2. Revised version of Fig. 2 in the original paper. The folding potential for the ${ }^{16} \mathrm{O}-{ }^{16} \mathrm{O}$ elastic scattering at $70 \mathrm{MeV} /$ nucleon. The real and imaginary parts of the potential are shown in the upper and lower panels, respectively. The solid (dashed) curve represents the result with (without) the $3 \mathrm{NF}$ effects. 

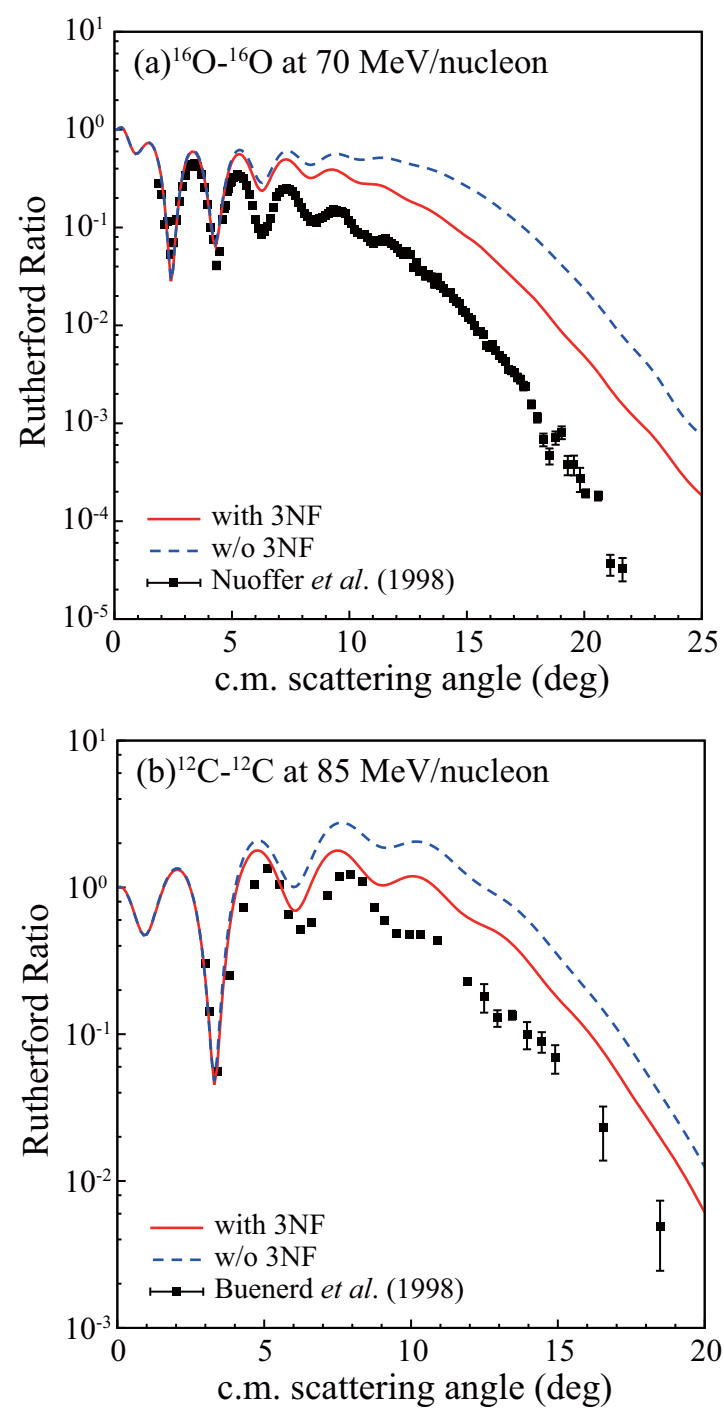

FIG. 3. Revised version of Fig. 3 in the original paper. The differential cross sections of (a) the ${ }^{16} \mathrm{O}-{ }^{16} \mathrm{O}$ elastic scattering at $70 \mathrm{MeV} / \mathrm{nucleon}$ and (b) the ${ }^{12} \mathrm{C}-{ }^{12} \mathrm{C}$ elastic scattering at $85 \mathrm{MeV} /$ nucleon as a function of the scattering angle in the center-of-mass system. The solid (dashed) line corresponds to the result of Melbourne interaction with (without) the $3 \mathrm{NF}$ corrections. The experimental data are taken from Refs. [3,4].

[1] M. Kohno, preceding Erratum, Phys. Rev. C 96, 059903 (2017).

[2] M. Kohno, Phys. Rev. C 88, 064005 (2013).
[3] F. Nuoffer et al., Nuovo Cimento A 111, 971 (1998).

[4] M. Buenerd et al., Phys. Lett. B 102, 242 (1981). 